\title{
MORITA EQUIVALENT SEMIGROUPS OF QUOTIENTS
}

\author{
JOHN K. LUEDEMAN
}

\begin{abstract}
Let $S$ be a monoid and ${ }_{S} M$ and ${ }_{S} N$ be retracts of each other. We show that $\operatorname{End}_{S}(M)$ and $\operatorname{End}_{S}(N)$ are Morita equivalent. Using this result, we show that if $A$ and $B$ are Morita equivalent monoids, then their semigroups of quotients are Morita equivalent.
\end{abstract}

Let $A$ and $B$ be monoids with 0 and let ${ }_{A}$ Tl (resp. ${ }_{B}$ T) denote the category of unitary left $A$-systems (resp. $B$-systems). The monoids $A$ and $B$ are Morita equivalent if there are covariant functors $F:{ }_{B} \mathfrak{N} \rightarrow_{A} \mathfrak{N}$ and $G:{ }_{A} \mathfrak{N} \rightarrow_{B} \mathfrak{N}$ with $F \cdot G \simeq 1_{A^{x x}}$ and $G \cdot F \simeq 1_{B}$. Knauer [3] (and independently, Banaschewski [1]) has shown that when $A$ and $B$ are Morita equivalent, there is an indecomposable projective generator $Q$ in ${ }_{B}$ 㲙 with $Q \simeq B b$ where $b^{2}=b \in B$ and $1,1^{\prime} \in B$ with $b 1=1$ and $1^{\prime} 1=e$, the identity of $B$. Moreover, $A \simeq F(Q), A \simeq \operatorname{Hom}_{B}(Q, Q) \simeq$ $b B b$ and $G(X)={ }_{B} Q_{A} \otimes X$.

In the following section, we show that when $S$ is a monoid with 0 and ${ }_{s} M,{ }_{s} N \in{ }_{s} \mathfrak{T}$ are retracts of each other, the endomorphism semigroups $\operatorname{End}_{s}(M)$ and $\operatorname{End}_{S}(N)$ are Morita equivalent. In $\S 2$, we use this result to show that when $A$ and $B$ are Morita equivalent monoids, then the McMorris quotient semigroups [7] $Q(A)$ and $Q(B)$ are Morita equivalent. This result was established in [5] for rings.

1. Retracts and Morita equivalence. Let $S$ be a monoid and ${ }_{s} M$ and ${ }_{s} N$ be left $S$-systems. Set $A=\operatorname{End}_{S}(M)$ and $B=\operatorname{End}_{S}(N)$; then $A$ and $B$ operate on the right of $M$ and $N$ respectively and so we have bi-systems ${ }_{S} M_{A},{ }_{S} N_{B},{ }_{A} \operatorname{Hom}_{S}(M, N)_{B}$ and ${ }_{B} \operatorname{Hom}_{S}(N, M)_{A}$.

Now we assume that the $S$-system $N$ is a retract of $M$ by means of the mappings $M \underset{\alpha}{\stackrel{\beta}{\rightleftarrows}} N$ with $\alpha \beta=1_{N}$. Define the mapping $\mu: \operatorname{Hom}_{S}(N, M) \otimes_{A} \operatorname{Hom}(M, N) \rightarrow B$ by $\mu(f \otimes g)=f g$. Then for $b \in B, \mu(b \alpha \otimes \beta)=b$ so $\mu$ is onto. Moreover if $\mu(f \otimes g)=\mu(x \otimes y)$, then $f g=x y$ so $f \otimes g=f \otimes g \alpha \beta=f g \alpha \otimes \beta=x y \alpha \otimes \beta=$ $x \otimes y \alpha \beta=x \otimes y$. Thus $\mu$ is an isomorphism of $(B, B)$-systems.

Next define $\psi: \operatorname{Hom}_{S}(M, N) \rightarrow \operatorname{Hom}_{A}\left(\operatorname{Hom}_{S}(N, M), A\right)$ by $\psi(f)(g)=f g$ and $\psi^{\prime}: \operatorname{Hom}_{S}(N, M) \rightarrow \operatorname{Hom}_{A}\left(\operatorname{Hom}_{S}(M, N), A\right)$ by $\psi^{\prime}(g)(f)=f g$. Then $\psi$ is an $(A, B)$-homomorphism and $\psi^{\prime}$ is a $(B, A)$-homomorphism. Using $\psi$ we have a commutative diagram

Received by the editors May 2, 1979 and, in revised form, September 29, 1979; presented to the Society, January 5, 1980 at San Antonio, Texas.

1980 Mathematics Subject Classificaton. Primary 20M50.

Key words and phrases. Semigroup of quotients, Morita equivalence.

(c) 1980 American Mathematical Society 0002-9939/80/0000-0505/\$02.00 


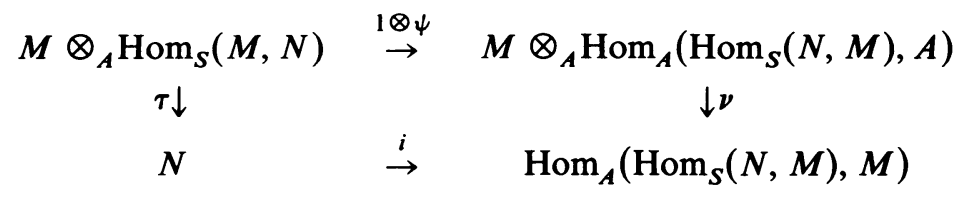

FIGURE 1

where $\tau(m \otimes f)=m f, i(n)(g)=n g$ and $\nu(m \otimes h)(g)=m \cdot(h(g))$ respectively. These are all $(A, B)$-homomorphisms.

THEOREM. If ${ }_{s} N$ is a retract of ${ }_{S} M$, then:

(1) $\psi$ and $\psi^{\prime}$ are isomorphisms.

(2) All homomorphisms in Figure 1 are isomorphisms.

(3) $\operatorname{Hom}_{S}(M, N)$ is a cyclic projective left $A$-system and a generator as a right $B$-system.

(4) $\operatorname{End}_{A}\left(\operatorname{Hom}_{S}(M, N)\right) \simeq B$ and $\operatorname{End}_{A}\left(\operatorname{Hom}_{S}(N, M)\right) \simeq B$.

Proof. (1) The inverse of $\psi$ is $\psi^{-1}(t)=t(\alpha) \beta \in \operatorname{Hom}_{S}(M, N)$.

(2) Define $k: \operatorname{Hom}_{A}\left(\operatorname{Hom}_{S}(N, M), M\right) \rightarrow M \otimes_{A} \operatorname{Hom}_{S}(M, N)$ by $k(f)=f(\alpha) \otimes$ $\beta$. Then all composition maps from one term to itself are the identity.

(3) ${ }_{A} \operatorname{Hom}_{S}(M, N)$ is a retract of $A$ by $f \in \operatorname{Hom}_{S}(M, N) \mapsto f \alpha \in \operatorname{Hom}_{S}(M, M)$ $=A$ and $g \in A=\operatorname{Hom}_{S}(M, M) \mapsto g \beta \in \operatorname{Hom}_{S}(M, N)$. Likewise, $\operatorname{Hom}_{S}(N, N)$ $=B$ is a retract of $\operatorname{Hom}_{S}(M, N)_{B}$ by $f \in \operatorname{Hom}_{S}(N, N) \mapsto \beta f \in \operatorname{Hom}_{S}(M, N)$ and $g \in \operatorname{Hom}_{s}(M, N) \mapsto \alpha g \in \operatorname{Hom}_{S}(N, N)$. Thus, $\operatorname{Hom}_{S}(M, N)$ is a cyclic projective left $A$-system and a generator as a right $B$-system.

(4) Let $\operatorname{Hom}_{S}(M, N)=A t$ and since $A t$ is a retract of $A$, we can regard at $\neq b t$ for $a \neq b$. Now map $\operatorname{Hom}_{A}(A t, A) \otimes_{A} A t$ to $\operatorname{Hom}_{A}(A t, A t)$ by $f \otimes_{A} b t \mapsto[f, b t]$ where $(a t)[f, b t]=(a t) f b t$. This mapping is onto since each $g: A t \rightarrow A t$ is determined by its image $g(t)=a_{g}$, so that if $h: A t \rightarrow A$ is given by $h(t)=a_{g}$, then $[h, t]=g$. To see that this mapping is injective, let $[h, b t]=[k, c t]$ so that $(t h) b t=$ $(t k) c t$, or $(t h) b=(t k) c$ or $(t) h b=(t) k c$ and so $h b=k c$. Thus $h \otimes b t=h b \otimes t=$ $k c \otimes t=k \otimes c t$ and so $\operatorname{Hom}_{A}\left(\operatorname{Hom}_{S}(M, N), A\right) \otimes_{A} \operatorname{Hom}_{S}(M, N)$ is isomorphic to $\operatorname{End}_{A}\left(\operatorname{Hom}_{S}(M, N)\right)$. Define $F:{ }_{B} \mathfrak{N} \rightarrow_{A} \mathfrak{N}$ by $F(X)=\operatorname{Hom}_{S}(M, N) \otimes_{B} X$ and

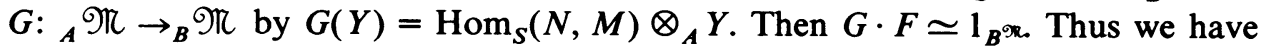
the following

TheOREM. Let ${ }_{S} M$ and ${ }_{S} N$ be retracts of each other. Then (1) $\operatorname{Hom}_{S}(M, N)$ is a cyclic projective generator both as a left $A$-system and a right $B$-system, and (2) $F$ and $G$ are inverse equivalences between ${ }_{A} \Re$ and ${ }_{B} \Re$.

2. Semigroups of quotients. A special left quotient filter $\Sigma$ of a monoid $B$ is a nonempty collection of left ideals of $B$ saitsfying

Q1. $I, J \in \Sigma, f \in \operatorname{Hom}_{B}(I, B) \Rightarrow f^{-1}(J) \in \Sigma$;

Q2. if $J \in \Sigma, I a^{-1}=\{s \in B \mid s a \in I\} \in \Sigma$ for all $a \in J$, then $I \in \Sigma$; and

Q3. $B \in \Sigma$.

For each left $B$-system $M$ we define the torsion congruence $\tau_{M}$ by $m \tau_{M} n$ if there is $I \in \Sigma$ with $b m=b n$ for all $b \in I$. $M$ is strongly torsion free if $\tau_{M}$ is the identity, 
and $M$ is torsion if $\tau_{M}=\omega . M$ is $\Sigma$-injective if whenever $C$ is a subsystem of $D$ and $D / C$ is torsion, then each $B$-homomorphism $f: C \rightarrow M$ has an extension $f: D \rightarrow M$. Each strongly torsion free $B$-system has a unique (up to isomorphism over $M$ ) extension $E_{\Sigma}(M)$ which is the minimal $\Sigma$-injective extension of $M . E_{\Sigma}(M)$ is called the $\Sigma$-injective hull of $M$.

Given a special left quotient filter $\Sigma$, let $\mathscr{B}=\cup_{I \in \Sigma} \operatorname{Hom}_{B}(I, B)$ and let $\theta$ be a congruence on $\beta$ given by $f \theta g$ if there is some $J \in \Sigma$ on which $f$ and $g$ are both defined and agree. Then $Q_{\Sigma}(B)=\Re / \theta$ with the operation of functional composition $f g: g^{-1}\left(D_{f}\right) \cap D_{g} \rightarrow B$ forms a monoid. Moreover, $Q_{\Sigma}(B)$ is the $\Sigma$-injective hull of $B$ if $B$ is strongly torsion free. For other definitions and concepts we refer the reader to [4].

Let $A$ and $B$ be Morita equivalent monoids. We use the following notation to describe this situation.

(1) $F:{ }_{B} \mathfrak{T} \rightarrow_{A} \mathfrak{R}$ and $G:{ }_{A} \mathfrak{N} \rightarrow{ }_{B} \mathfrak{N}$ are inverse category equivalences.

(2) ${ }_{A} P_{B}$ is an $(A, B)$-bisystem which is a left $A$ and right $B$ progenerator (i.e., cyclic projective generator).

(3) ${ }_{B} Q_{A}$ is a $(B, A)$-bisystem which is a left $B$ and right $A$ progenerator.

(4) $F_{-}=P \otimes_{B-}$ and $G_{-}=Q \otimes_{A_{-}}$.

(5) $A \simeq \operatorname{End}_{B}(P) \simeq \operatorname{End}_{B}(Q)$ and $B \simeq \operatorname{End}_{A}(Q) \simeq \operatorname{End}_{A}(P)$.

(6) ${ }_{B} Q \simeq \operatorname{Hom}_{B}(P, B)$ and $A \simeq \operatorname{Hom}_{A}(Q, A)$.

We have over-determined our equivalence. For $A$ and $B$ to be Morita equivalent it is necessary and sufficient that $A \simeq \operatorname{End}_{B}(P)$ for some progenerator $P_{B}$ [5]. (Although [5] deals only with the additive case, since $P_{B}$ is a cyclic projective generator, simple modifications of the existing proofs suffice to justify that $A \simeq$ End $_{B}(P)$ suffices for $A$ and $B$ to be Morita equivalent.) Since ${ }_{B} Q$ is a progenerator and $B$ is a monoid, $B$ and $Q$ are retracts of each other. Without loss of generality, since $B$ is a monoid, $Q$ is indecomposable [3].

Let $\Sigma$ be a special left quotient filter on $B$ and $\mathcal{T}(B)$ be the class of all $\Sigma$-torsion $B$-systems. $\Sigma$ uniquely determines and is determined by $\mathscr{T}(B)$, where $\mathscr{T}(B)$ is special in the sense that if $f \in \operatorname{Hom}(M, N)$ is 0 -restricted (i.e., $\left.f^{-1}(0)=\{0\}\right)$ and $N \in \mathcal{T}(B)$ then $M \in \mathcal{T}(B)[4]$. Let $\mathcal{T}(A)=\left\{N \in{ }_{A} \mathfrak{T} \mid G(N) \in \mathcal{T}(B)\right\}$. Since $G$ is an equivalence, $\mathcal{T}(A)$ is closed under quotients, disjoint unions, and extensions and so is a torsion class for ${ }_{A} \mathfrak{T}$. Moreover, $\mathscr{T}(A)$ is a special torsion class, for if $f \in \operatorname{Hom}_{A}(M, N)$ is 0-restricted and $N \in \mathcal{T}(A)$, then $G(N) \in \mathcal{T}(B)$ and $G(f): G(M) \rightarrow G(N)$. Suppose $G(K) \subset G(M)$ with $G(f)(G(K))=0$; then since $G$ is an equivalence, $f(K)=0$ so $K=0$ since $f$ is 0 -restricted. Thus $G(K)=0$ and $G(f)$ is 0-restricted. Since $\mathscr{T}(B)$ is a special torsion class, $G(M) \in \mathscr{T}(B)$ so $M \in \mathcal{T}(A)$. Thus $\mathcal{T}(A)$ is special and determines a unique special left quotient filter $\Gamma$ on $A$ by $\Gamma=\{J \mid G(A / J) \in \mathcal{T}(B)\}$. Thus we have

Theorem. $\Gamma=\{J \mid A / J \in \mathcal{T}(A)\}$ is a special left quotient filter on $A$.

Now let $M \in{ }_{B} \Re$; then since $F$ and $G$ are inverse equivalences, $M$ is $\Sigma$-injective if and only if $F(M)$ is $\Gamma$-injective. This yields the following

Proposition. $F\left(E_{\Sigma}(M)\right)=E_{\Gamma}(F(M))$. 
Since ${ }_{B} Q$ is a finitely generated projective generator, ${ }_{B} Q$ and ${ }_{B} B$ are retracts of each other. Knauer [3] (and independently Banaschewski [1]) has shown that $Q \simeq B b$ where $b^{2}=b \in B$. Now if $B$ is strongly torsion free, the isomorphism between $Q$ and $B b$ shows that $Q$ is strongly torsion free. Then by the definition of $\Gamma$ and the fact that $F$ and $G$ are category equivalences, $A$ is strongly torsion free.

Now since ${ }_{B} Q$ and ${ }_{B} B$ are retracts of each other, $E_{\Sigma}(Q)$ and $E_{\Sigma}(B)$ are retracts of each other. Since $F(Q) \simeq{ }_{A} A$ we have

$$
\operatorname{End}_{B}\left(E_{\Sigma}(Q)\right) \simeq \operatorname{End}_{A}\left(F\left(E_{\Sigma}(Q)\right)\right) \simeq \operatorname{End}_{A}\left(E_{\Gamma}(F(Q))\right) \simeq \operatorname{End}_{A}\left(E_{\Gamma}(A)\right)
$$

but $Q_{\Sigma}(B) \simeq \operatorname{End}_{B}\left(E_{\Sigma}(B)\right)$ and $Q_{\Gamma}(A) \simeq \operatorname{End}_{A}\left(E_{\Gamma}(A)\right) \simeq \operatorname{End}_{B}\left(E_{\Sigma}(Q)\right)$.

Let $M=E_{\Sigma}(Q)$ and $N=E_{\Sigma}(B)$; then from the last section we see that $Q_{\Sigma}(B)$ and $Q_{\Gamma}(A)$ are Morita equivalent and $\operatorname{Hom}_{B}\left(E_{\Sigma}(Q), E_{\Sigma}(B)\right)$ is a cyclic projective generator both as a left $Q(A)$-system and right $Q(B)$-system.

Since $P \otimes_{B} E_{\Sigma}(B)$ is $\Gamma$-injective and $E_{\mathrm{\Gamma}}(A) / A \in \mathcal{T}(A)$,

$$
\operatorname{Hom}_{A}\left(A, P \otimes_{B} E_{\Sigma}(B)\right) \simeq \frac{\operatorname{Hom}_{A}\left(E_{\Gamma}(A), P \otimes_{B} E_{\Sigma}(B)\right)}{\operatorname{Hom}_{A}\left(E_{\Gamma}(A) / A, P \otimes_{B} E_{\Sigma}(B)\right)},
$$

but since $P \otimes_{B} E_{\Sigma}(B)$ is strongly torsion free,

$$
\operatorname{Hom}_{A}\left(E_{\Gamma}(A) / A, P \otimes_{B} E_{\Sigma}(B)\right)=0,
$$

and $\operatorname{Hom}_{A}\left(A, P \otimes_{B} E_{\Sigma}(B)\right)$ and $\operatorname{Hom}\left(E_{\Gamma}(A), P \otimes_{B} E_{\Sigma}(B)\right)$ are isomorphic as right $Q_{\Sigma}(B)$-systems. Thus

$$
\begin{aligned}
\operatorname{Hom}_{B}\left(E_{\Sigma}(Q), E_{\Sigma}(B)\right) & \simeq \operatorname{Hom}_{A}\left(E_{\Gamma}(A), P \otimes_{B} E_{\Sigma}(B)\right) \simeq \operatorname{Hom}_{A}\left(A, P \otimes_{B} E_{\Sigma}(B)\right) \\
& \simeq P \otimes_{B} E_{\Sigma}(B) \simeq P \otimes_{B} Q_{\Sigma}(B) .
\end{aligned}
$$

Thus we have proved the following

Theorem. Let $\Sigma$ be a special left quotient filter on the monoid $B$ and let $A$ and $B$ be Morita equivalent monoids. If $\Gamma$ is the corresponding special left quotient filter on $A$, then $Q_{\Gamma}(A)$ and $Q_{\Sigma}(B)$ are Morita equivalent. Moreover, in the situation above, $P \otimes_{B} Q_{\Sigma}(B)$ is right $Q_{\Sigma}(B)$-cyclic projective generator and

$$
Q_{\Gamma}(A) \simeq \operatorname{End}_{Q_{\Sigma}(B)}\left(P \otimes_{B} Q_{\Sigma}(B)\right) .
$$

REMARK. I would like to thank the referee for his helpful comments and for advising me of reference [1].

\section{BIBLIOGRAPHY}

1. B. Banaschewski, Functors into categories of M-sets, Abh. Math. Sem. Univ. Hamburg 8 (1972), 49-64.

2. K. Hirata, Some types of separable extensions of rings, Nagoya Math. J. 33 (1968), 107-115.

3. U. Knauer, Projectivity of acts and Morita equivalence of monoids, Semigroup Forum 3 (1972), 359-370.

4. J. Luedeman, Torsion theories and semigroups of quotients, Tech. Rep. 297, Clemson University, Clemson, S. C.

5. B. Pareigis, Categories and functors, Academic Press, New York, 1970.

6. D. Turnidge, Torsion theories and rings of quotients of Morita equivalent rings, Pacific J. Math. 37 (1971), 225-234.

7. H. J. Weinert, On $S$-sets and semigroups of quotients, Semigroup Forum 19 (1980), 1-78.

Department of Mathematical Sciences, Clemson University, Clemson, South Carolina 29631 